\title{
A New Fueling Process in a Weak Bar
}

\author{
Keiichi Wada \\ Center for Information Processing Education, Hokkaido University, \\ Sapporo 060, Japan
}

Asao Habe

Department of Physics, Hokkaido University, Sapporo 060, Japan

\begin{abstract}
A massive gaseous disk in the central region of a galaxy sensitively responds to a weakly distorted potential, and a large amount of gas can be fed into within $1 / 20$ of the core radius of the potential in several $10^{7} \mathrm{yr}$. The ILRs, the dissipative nature of the gas, and the selfgravity of the gas are essential for triggering this effective fueling. We also found that a counterrotating gaseous core can be formed as a result of the fueling. Our result suggests that the merger of galaxies is not the only way to form the observed counterrotating core in galaxies.
\end{abstract}

\section{Introduction}

It is generally accepted that nuclear activities in galaxies, such as starbursts, result from feeding of the gas into a nuclear region. Many studies in the last two decades have revealed the fueling process from $10 \mathrm{kpc}$ to several hundred pc from the center. A stellar bar, which is formed by gravitational instability of a stellar disk or triggered by close-encounters of galaxies, is the most probable cause of the fueling. Due to angular momentum exchange between the gas and the bar, a large amount of gas can be accumulated near to the ILR, which is usually located at $\sim 1 \mathrm{kpc}$ from the center. Numerical simulations, however, have shown that the bar cannot force the gas to fall beyond the ILR. To break the 'ILR barrier', self-gravity of the gas is essential (Fukunaga \& Tosa 1991; Wada \& Habe 1992; Elmegreen 1994). Gravitational instability followed by collapse of the extremely elongated ILR ring results in a rapid mass accretion, $\sim 10^{9} M_{\odot}$ in $5 \times 10^{8} \mathrm{yr}$ to several hundred pc. However, fueling from several hundred pc to the central 1 pc would need a different mechanism.

Here we have investigated dynamical evolution of a massive gaseous disk in a central region of a galaxy with weak distortion. Massive gas disks should have been common in the galaxy-formation era as well as in nearby starburst galaxies. Recent near-infrared observations have often revealed weak bar-like structures in the central regions of normal spiral galaxies. By two dimensional hydrodynamical simulations taking into account the self-gravity of the gas, we have found a new fueling mechanism from kpc scales to several tens of pc, which is induced by a weak bar and the self-gravity of the gas. 


\section{Numerical Models and Method}

Gas disks are evolved in a weakly distorted bulge of a galaxy, which is represented by a non-axisymmetric potential, $\Phi_{1}(R, \theta)=\varepsilon_{0} \frac{a R^{2}}{\left(R^{2}+a^{2}\right)^{3 / 2}} \Phi_{0} \cos 2 \theta$, where $\varepsilon_{0}$ is a parameter which represents strength of the bar potential relative to the disk component. We have explored models with $\varepsilon_{0}=0.0,0.05,0.1$ and 0.2 . Since a maximum ratio of the non-axisymmetric part to the axisymmetric one is $\max \left(\left|\Phi_{1}(R)\right| /\left|\Phi_{0}(R)\right|\right)=0.385 \varepsilon_{0}$, the bars are very weak. In our model, if $0.005<\Omega_{\mathrm{b}}<0.026 \mathrm{~km} \mathrm{~s}^{-1} \mathrm{pc}^{-1}$, there are two ILRs for a ratio of gas mass to the dynamical mas of $f_{\mathrm{g}}=0.10$ inside the initial gas disk (the radius is 2.0 ), whereas there is one ILR at the vicinity of the center if $\Omega_{\mathrm{b}}>0.026$, and no ILRs for $\Omega_{\mathrm{b}}<0.005$.

Gas dynamics is calculated by SPH. The total number of SPH particles is $10^{4}$. The particles are randomly distributed in a disk with $R_{\text {gas }}=2.0 \mathrm{kpc}$ at $t=0$. The initial rotational velocity is given in order to balance centrifugal force caused by the axisymmetric gravitational potential, since the distorted potential is very weak. We assume that gas is isothermal (the sound velocity is 0.1 ).

The range of $F_{\mathrm{g}}$ is taken to be $0.03-0.30$. The total dynamical mass inside the gas disk, $M_{\text {dyn }}\left(R<R_{\text {gas }}\right)$ is $2.3 \times 10^{10} M_{\odot}$.

The equation of motion of the $i$-th SPH particle is

$$
\frac{d^{2} r_{i}}{d t^{2}}=-\nabla\left(\Phi_{\mathrm{axi}}+\Phi_{\mathrm{bar}}\right)-\nabla \Phi_{\mathrm{gas}}-\frac{1}{\rho_{i}} \nabla\left(P_{i}+q_{i}\right),
$$

where $\Phi_{\text {gas }}, P_{i}$ and $q_{i}$ are the gravitational potential of gas, the gas pressure, and the artificial viscosity, respectively.

The gravitational potential of the gas is calculated by solving Poisson's equation, $\Delta \Phi_{\text {gas }}=4 \pi G \rho_{\text {gas }}$. To solve this, we use the FFT method with $256 \times 256$ Cartesian grids, and the size of the grid is $0.05 \mathrm{kpc}$.

\section{Results}

We have found extremely non-axisymmetric gaseous inflow in most models. Figure 1 shows typical evolution of the gas disk in the co-rotating frame of the bar. A dense linear structure, which inclines by about $30^{\circ}$ with respect to the major axis of the distorted potential, is formed at the center in the first $T \sim 1.510^{7}$ yr. The linear dense region becomes denser with time, and the gas in the dense region falls toward the center. As a result, an extremely dense core is formed at the center. The size of the core is typically less than 0.1 and the core contains $\sim 1 / 3$ of the total gas.

In the early stage of the evolution, the gas moves on elliptic orbits oriented by $30^{\circ} \sim 45^{\circ}$ with respect to the oval potential, and the distortion of the orbits becomes strong with time. Eventually shocks are generated along the major axis of the elliptical orbits. When the gas on elongated orbits rushes into the shocked region, the orbits drastically change and the gas falls toward the center. The typical accretion rate is about $30 M_{\odot} \mathrm{yr}^{-1}$.

We found in some models that rotation of the gaseous core in the center is in an opposite sense with respect to the outer gas and the galactic rotation. 


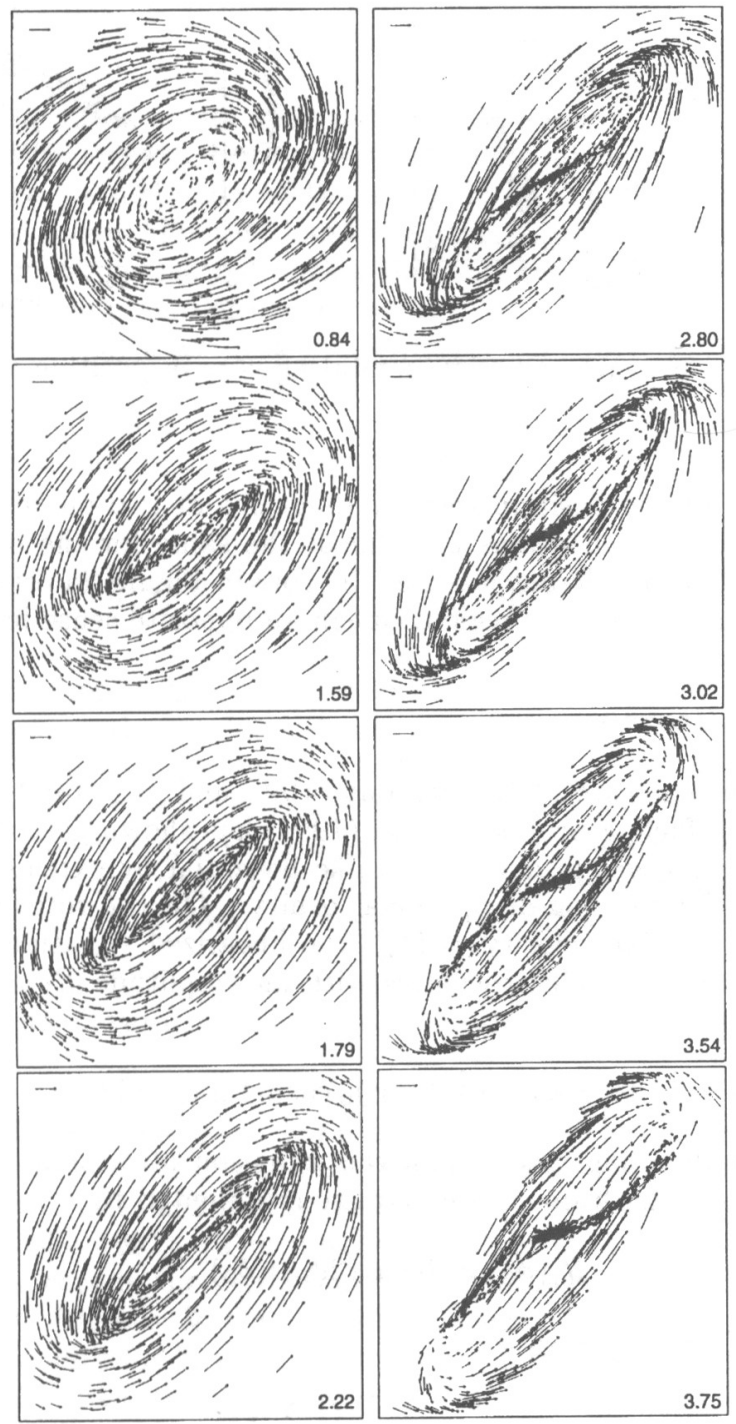

Figure 1. Gaseous velocity vectors in a model $\left(\Omega_{b}=0.02\right.$ and $\varepsilon_{0}=$ $0.2)$ are shown. Unit vector $\left(V=200 \mathrm{~km} \mathrm{~s}^{-1}\right)$ is shown at the upper left corner. Major axis of the bar horizontally is fixed on each frame. Time is shown at the right bottom corner. Frame size is $4.8 \times 4.8 \mathrm{kpc}$. 

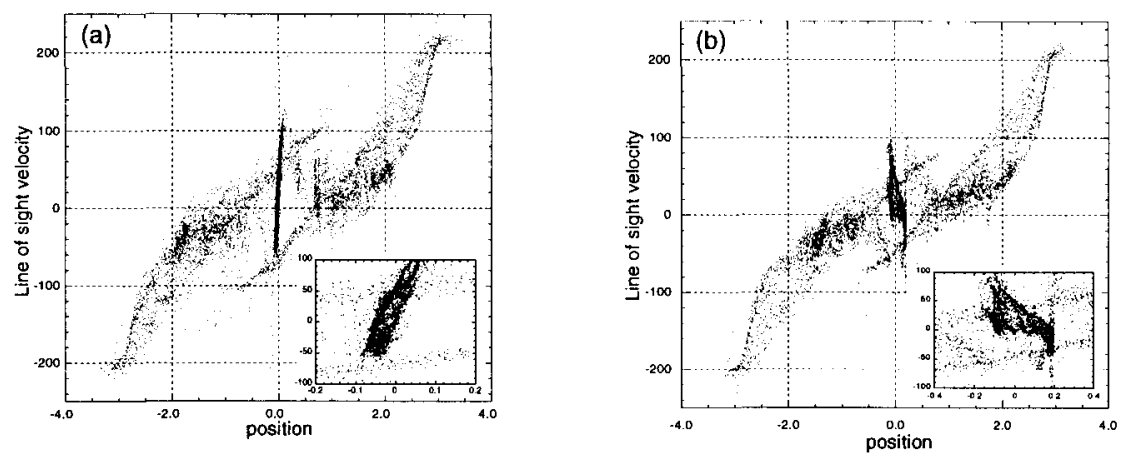

Figure 2. Normal and counterrotating core in position-velocity diagrams.

Figure $2 \mathrm{a}$ and $2 \mathrm{~b}$ are position-velocity diagrams of two models in the inertial frame. Although the diagrams are almost the same for the outer region, the nuclear cores show a different feature: the core in Figure 2a rotates in the same sense as the gas in the outer region, but in an opposite sense in Figure 2b.

\section{Physical Reason}

The dissipational nature of the gaseous orbits and self-gravity of the gas are essential for the fueling process. Wada (1994) has shown gaseous behavior of a non-self-gravitating gas in a weak bar-like potential by extending the epicycle approximation for the non-dissipational orbits. ${ }^{1}$ On the rotating frame of the bar potential (angular velocity $=\Omega_{\mathrm{b}}$ ), the linearized equation of motion of a particle with friction, which oscillates with an amplitude $R_{1}$ around a guiding center at $R_{0}$, is

$$
\ddot{R}_{1}+2 \lambda \dot{R}_{1}+\kappa_{0}^{2} R_{1}=f_{0} \cos 2\left(\Omega_{0}-\Omega_{\mathrm{b}}\right) t
$$

where $\lambda$ is the damping rate. From this equation of motion, 'damped' closed orbits on the rotating frame of the bar are represented by

$$
R_{1}\left(\phi_{0}\right)=B \cos \left(2 \phi_{0}+\delta_{0}\right)
$$

The phase-shift, $\delta_{0} \equiv \delta\left(R_{0}\right)$, is

$$
\delta_{0}=\arctan \left[\frac{2 F \Lambda}{F^{2}-1}\right],
$$

where

$$
F \equiv 2\left(\Omega_{0}-\Omega_{\mathrm{b}}\right) / \kappa_{0}, \Lambda \equiv \lambda / \kappa_{0}
$$

\footnotetext{
${ }^{1}$ Lindblad \& Lindblad (1994) reached almost the same conclusion.
} 
The important point is that the phase-shift $\delta_{0}$ is always negative if $F>0$, and $\delta_{0}=-\pi / 2$ when $F^{2}=1$, that is, at the Lindblad resonances. The phase delay of the damped oscillation appears as a leading shift of the oval orbits with respect to the bar potential if $B>0$. As can be seen in Figure 3 in Wada (1994), the amplitude $B$ is positive $\left(R<R_{\mathrm{CR}}\right.$ ). Therefore the oval gas orbits have always a leading shift with respect to the bar potential inside the inner ILR. In fact, the shift is $30^{\circ} \leq \Delta \leq 45^{\circ}$ in the hydrodynamical simulations. Consequently the gas on the elongated orbits near to the ILRs loses its angular momentum (see Figure 5 in Wada 1994). Both self-gravity and the angular momentum loss of the gas enhance the distortion of the oval orbits. Therefore the formation of the linear shocked region (Figure 1) and the nuclear inflow of the gas inevitably occur in a self-gravitating gas disk within the inner two ILRs of three ILRs, even if the distortion of the potential is very weak.

We have found that a gas disk in a stronger bar more rapidly evolves and a larger amount of the gas can be fed into the center in a more early phase. This fact implies that the torque exerted from the non-axisymmetric potential determines the evolution of the system. The torque, $\tau(R, \theta)$, caused by the bar potential is

$$
\tau(R, \theta)=-2 \varepsilon a \alpha \frac{R^{2}}{\left(R^{2}+a^{2}\right)^{2}} \sin 2 \theta
$$

and has the extremum at $R=a$ and $\theta= \pm \pi / 4, \pm 3 \pi / 4$. If we assume the gas rotates anti-clockwise, the gas loses its angular momentum in the first and the third quadrant. Especially, the gas in the linear dense region leading by $45^{\circ}$ to the bar potential most effectively looses its angular momentum. We have found that if the bar is slow enough, the gas in the dense region can easily change the sign of its angular momentum. Therefore the counterrotating gaseous core is a natural consequence of the resonant structure in a self-gravitating gas disk.

\section{Origin of the Counter Rotating Core in Galaxies}

Kinematically decoupled stellar cores or counterrotating gaseous cores are often found in elliptical and S0 galaxies. For example, IC 1459 has one of the strongest counterrotating components of any observed elliptical galaxy (Forbes, Franx \& Illingworth 1994). NGC 7252 has a counterrotating molecular gas disk (Wang, Schweizer \& Scoville 1992). The most widely accepted model for producing a counterrotating core is the retrograde merger of a compact elliptical galaxy (Kormendy 1984; Balcells \& Quinn 1990). Our numerical results, however, give another possibility for forming the counterrotating gaseous or stellar cores. We need an ovally distorted potential and a massive gaseous disk inside the turnover radius of the rotation curve. The rotating non-axisymmetric potential must be generated in most elliptical and S0 galaxies, which have a triaxial stellar distribution. Gaseous disks are not uncommon in early-type galaxies, and must have existed in ellipticals if they were formed from mergers of gas rich spiral galaxies. Although star-formation should properly be taken into account in our numerical simulations, the observed stellar counterrotating cores could be formed from the gaseous counterrotating cores. 


\section{References}

Balcells, M. \& Quinn, P. J. 1990, ApJ, 361, 381

Elmegreen, B. G. 1994, ApJ, 425, L73

Forbes, D. A., Franx, M., \& Illingworth, G. D. 1994, ApJ, 428, L49

Fukunaga, M. \& Tosa, M. 1991, PASJ, 43, 469

Kormendy, J. 1984, ApJ, 287, 577

Lindblad, P. O. \& Lindblad, P. A. B. 1994, in The Gaseous and Stellar Disks of the Galaxy, I. R. King, San Francisco: ASP, 29

Rubin, V. C. 1994, AJ, 108, 456

Wada, K. 1994, PASJ, 46, 165

Wada, K. \& Habe, A. 1992, MNRAS, 258, 82

Wang, Z., Schweizer, F., \& Scoville, N. Z. 1992, ApJ, 396, 510

\section{Discussion}

J. Kenney: Does your simulation need to have gas originally inside the IILR in order to cause the inflow?

K. Wada: Yes. If there is no gas or the gas density is very low inside the IILR, the gas forms an oval ring outside the IILR, and the rapid fueling does not occur until the ring becomes gravitationally unstable and collapses. Dissipation of the gas is essential for the oval orbits with a leading shift. The linear shocked region, in which the rapid inflow occurs, is originated in these peculiar orbits inside the IILR. The rapid inflow does not also occur in a massive and highly gravitationally unstable gas disk, because dissipation does not effectively work in a few-body clump system.

G. Galletta: The nuclear gas counterrotation you predict extends $100 \mathrm{pc}$. At the distance of the Virgo cluster, this corresponds to $1^{\prime \prime}$, which is not observable, but shows an expansion of the line-width. This phenomenon has not been observed.

K. Wada: Conditions for producing the counterrotating gas cores might be severe in most galaxies. In our simulation, the fraction of the gas mass to the dynamical mass should be in the range $\sim 0.03-0.12$, and the rotating speed of the bar should be around the peak of $\Omega_{0}-\kappa_{0} / 2$. I am not sure whether these conditions are satisfied in galaxies in Virgo, but it is not a mystery if there are no galaxies with a counterrotating core in the Virgo cluster.

Z. Tsvetanov: There are a couple of HST programs that reveal $\sim 100 \mathrm{pc}$ scale gaseous disks in elliptical galaxies. In the few cases that have been studied in more detail, however, the mass estimates are in the range a few $10^{4-6} M_{\odot}$. This is by three orders of magnitude lower than your prediction of $10^{8-9} M_{\odot}$. Do you have a comment on this?

K. Wada: We did not take the star formation in our model. The very high accretion rate to the central small region would cause intense starbursts in the gas core. It is a possibility that massive star formation and supernova explosions blow out most of the gas core, or prevent the fueling. 